\title{
POLUIÇÃO ATMOSFÉRICA RELACIONADA ÀS DOENÇAS RESPIRATÓRIAS: Abordagem de abrangência em prol da prática clínica
}

\author{
Raísa Laisner FREGONEZI ${ }^{1 *}$ \\ João Carlos Bicego Gomes RIBEIRO ${ }^{2}$ \\ José Ribamar Moura SIMEÃO ${ }^{3}$ \\ Túlio Oliva CARVALHO \\ Laura Ferreira de Rezende FRANCO 5
}

\author{
${ }^{1-4}$ Discentes. \\ ${ }^{5}$ Mestre e Doutora pela Faculdade de Ciências Médicas UNICAMP e pós-doutora pela UNESP. laura@fae.br \\ * raisa.fregonezi@hotmail.com
}

Recebido em: 03/03/2015 - Aprovado em: 02/07/2015 - Disponibilizado em: 15/07/2015

\begin{abstract}
RESUMO
A urbanização e outras atividades humanas como a indústria, os transportes e até mesmo a agropecuária de forma mal planejada vêm causando grandes impactos ambientais, especialmente na atmosfera. As grandes quantidades de gases poluentes que são liberadas no ar podem acarretar problemas respiratórios para a população humana exposta a esses poluentes. Desenvolveu-se um estudo de revisão correlacionando o impacto respiratório causado por alguns poluentes na atmosfera terrestre. A hipótese, embasada em artigos científicos, diz respeito à grande quantidade de gases e outras substâncias lançadas pelo homem no ar, que podem trazer patologias associadas ao sistema respiratório humano. O trabalho em questão foi feito mediante a seleção e análise de alguns artigos e estudos coerentes com o tema escolhido, os quais foram encontrados nas bases de dados PubMed, Lilacs e Scielo. Foram comparadas as diversas populações e as substâncias poluentes apresentadas, além de ter sido observado que a exposição a uma atmosfera com alguns tipos de poluentes tais como: ácido sulfúrico, ozônio, dióxido de enxofre, e metacolina, gera o aparecimento de patologias respiratórias, como a reação alérgica e a asma. Ficou evidenciado que o lançamento dos poluidores na atmosfera aumenta a poluição do ar, que se encontra intimamente relacionada com o aparecimento das doenças respiratórias.
\end{abstract}

Palavras-chave: AR. POLUIÇÃO. RESPIRATÓRIO. ALERGIA. ASMA.

\section{AIR POLLUTION RELATED TO RESPIRATORY DISEASES: A comprehensive approach to clinical practice}

\begin{abstract}
Urbanization and other human activities such as industry, transport and even poorly planned form of agriculture are causing major environmental impacts, especially in the atmosphere. Large amounts of pollutants, which are released in the air can cause respiratory problems for the human population exposed to these pollutants. We developed a review study correlating the respiratory impact of some pollutants in the atmosphere. The hypothesis grounded in scientific articles reputed concerns as the large amount of gases and other substances released by man into the air, can bring diseases associated with human respiratory system. The work in question was made by the selection and analysis of some articles and studies consistent with the chosen theme, which were found in the databases PubMed, Lilacs and Scielo. The various population and presented polluting substances were compared, and it has been confirmed by tests that exposure to an atmosphere with some types of pollutants such as sulfuric acid, ozone, sulfur dioxide, and methacholine generates the onset of respiratory diseases such as asthma and allergic reaction. It was demonstrated that with the release of pollutants in the atmosphere, there is an increased in air pollution that is closely linked to an outbreak of respiratory diseases.
\end{abstract}

Keywords: AIR. POLLUTION. RESPIRATORY. ALLERGY. ASTHMA. 


\section{INTRODUÇÃO}

Este estudo está orientado para a prática da clínica médica, como um facilitador na busca por informações sobre a associação entre patologias respiratórias e poluição do ar, e se faz cada vez mais relevante por conta dos altos índices de poluição e decorrentes internações (Hernandez-Cadena, 2000) por problemas de saúde (Albuquerque de Castro, 2007) observadas nos últimos anos. Cabe ressaltar que sobre este tema ainda existem questões em aberto, que necessitam de maior aprofundamento e comprovação através de experimentos, como é o caso das inferências obtidas nos trabalhos de Schram-Bijkerk (2006), as quais não acordam com os trabalhos científicos anteriores que demonstram uma relação dose-resposta linear, enquanto os de Bijkerk demonstraram, com fatores que ainda precisam ser melhor estudados, uma relação em forma de sino. No entanto, são também perceptíveis algumas constatações presentes em grande parcela dos artigos e já fortemente fundamentadas, como a atopia (Castro, 2002), a susceptibilidade da população urbana às alergias respiratórias em detrimento da população rural, muito menos sensibilizada pelos alérgenos em decorrência do maior contato com os mesmos (Martinez, 1994), ordem de nascimento, alérgenos sazonais (Sunyer, 1997) e grau de obstrução brônquica (Utell, 1994). Todos estes fatores convergem para os testes experimentais em favor do aprimoramento das técnicas em prol de descobertas que estão longe de serem finalizadas, a respeito deste campo tão abrangente e, simultaneamente, minucioso, o das alergias respiratórias.

\section{MÉTODO}

Foram analisados nove artigos, nas bases de dados PubMed, Lilacs e Scielo. Para viabilizar a pesquisa, utilizaram-se as seguintes palavras-chaves: AR, POLUIÇÃO, RESPIRATÓRIO, ALERGIA, ASMA, combinadas aleatoriamente. Dessa maneira, foram selecionados artigos no período de 1988 a 2007, que atendessem aos objetivos propostos por este estudo. Nos diferentes artigos foram encontrados os métodos: questionário simples e curto para análise, exposição ao ácido sulfúrico e ao ozônio, exposição ao dióxido de enxofre, espirometria e testes com metacolina, históricos e prontuários de pacientes, históricos de pacientes que foram a óbito por doenças respiratórias e sensibilização avaliada pela medição de alérgeno específico e contra ácaros.

\section{RESULTADOS}

A tabela abaixo objetiva relacionar a análise dos diferentes resultados encontrados com respectivos fatores associados à poluição 


\section{atmosférica e aos agravantes das patologias}

\section{respiratórias.}

Tabela I - Descrição dos artigos selecionados nas bases de dados PubMed, Lilacs e Scielo, quanto aos autores, ao desenho de estudo, métodos, resultados e explicações para as inferências.
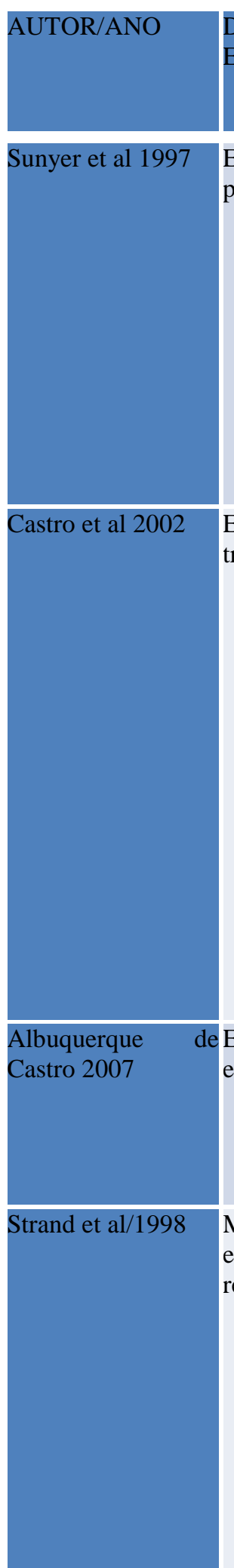

Sacramento oliveira 2011
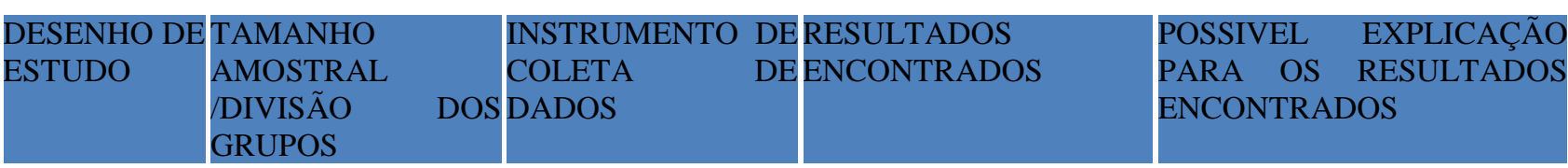

Estudo de 2646 sujeitos Foi desenvolvido umParticipantes do desafio Asma é mais frequente em prevalência responderam umquestionário simples e diferem significativamente pessoas de idade superior a 15 questionário curto para análise em área e sexo comparado anos e também aos sujeitos que respiratório e destes, respiratória via e-mailcom outros somentepossuem a doença em suas 1797 participaram de e telefone. Na entrevistados. A famílias. um desafio bronquial. segunda fase, $20 \%$ prevalência da asma A escolha foi através dos sujeitos foram começa a aumentar a partir de amostra selecionados para odos 15 anos. A forte randomizada da desafio de inalação de associação entre atopia e população entre 20 e metacolina. asma parental com asma 44 anos em 5 áreas da recorrente foi confirmada. Espanha.

Estudo Estudo comparativo A análise estatística Não foram observadas Houve maior sensibilização em transversal entre dois grupos de foi realizada com odiferenças estatisticamente São Paulo do que em Atibaia. 40 alunos de escolas teste do Qui-quadradosignificativas para os As populações estudadas públicas, uma em São e Mann-Whitney, sintomas pesquisados apresentaram o mesmo perfil Paulo e outra emconsiderando-se comoentre os grupos, que de sensibilização, com maior Atibaia, com média significantes os também mostraram-se número de testes positivos para de idade de dozevalores de $\mathrm{p}<0,05$. homogêneos em relaçãoBlomia tropicalis, anos. aos antecedentes pessoais Dermatophagoides e familiares de alergia. A pteronyssinus, poluição tem influência no Dermatophagoides farinae, desenvolvimento deAspergillus fumigatus e aeroalérgenos. Penicilium notatum. Os achados apoiam a hipótese de que a poluição presente na área urbana pode estar envolvida na maior sensibilização entre os escolares da região.

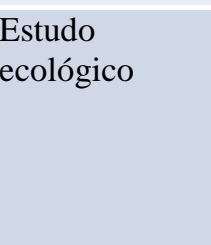

Modelo exposição repetida
Foram pesquisados Históricos 293.305 habitantes de prontuários Vitoria, Espírito pacientes. Santo. de 16 diagnosticados asma leve. pacientes A função pulmonar Resposta asmática

eO nível de poluição emOs casos de Vitória indicam deVitória é relativamente que há uma relação social com baixo, quando comparado os problemas respiratórios, isto com o nível de risco daé, em lugares mais pobres legislação brasileira e da existem mais problemas OMS. respiratórios.

foi Houve uma tendência $(\mathrm{p}=$ $0,07)$ para o aumento dos om(volume expiratóriosignificativamente forçado no primeiro aumentada após exposiçãosintomas noturnos de asma segundo (FEV1)) foi repetida a NO2 e após a exposição ao NO2 mais medido através de umalérgenos se comparada à alérgeno. Embora os efeitos espirômetro. exposição ao ar e sejam pequenos, os resultados alérgenos. indicam que uma breve exposição repetida a um nível ambiente de NO2 aumenta a resposta asmática das vias aéreas a uma dose de alérgeno assintomática. de Estudo ecológico séries temporais
261.413 habitantes de Históricos

de O maior número de óbitos As diferenças fisiológicas entre de Volta Redonda, Rio pacientes que foram a ocorreu em Junho, por ser homens e mulheres sugere uma de Janeiro. óbito por doenças mais frio, e emissões possível diferença na resposta a respiratórias. diárias de PM10 edeterminados níveis de 699 

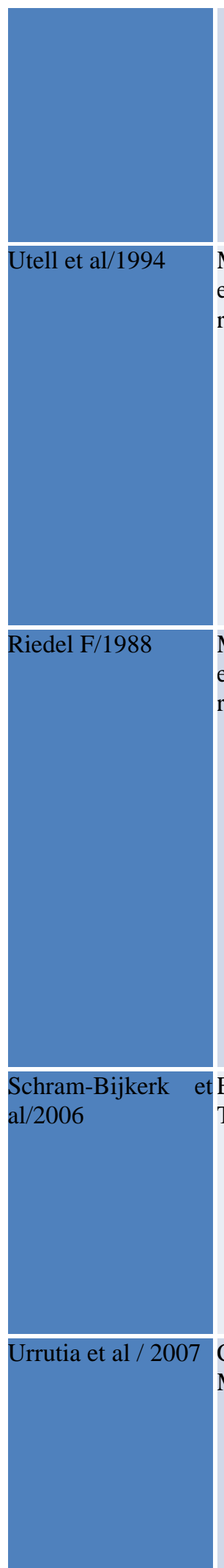

SO2 foram associadas exposição e composição com mortalidade porquímica dos poluentes, assim doenças respiratórias como o fator idade.

principalmente em

mulheres idosas, mas

mostraram um efeito

tardio também em

homens.

Modelo de Dois grupos de 30 Os indivíduos foram As principais conclusões Para examinar os efeitos exposição voluntários, não primeiro expostos a do estudo são: Não hátardios, foram medidos os repetida fumantes e de ambosácido sulfúrico efeitos sintomáticos ou testes de função pulmonar de os sexos, com idades(H2SO4) em aerossol fisiológicos significativos dois e quatro horas após a entre 18 e 45 anos,para sensibilizar as de exposição a qualquer exposição nos dias de ozônio. foram recrutados. vias aéreas ao ozônio. aerossol de ácido ou de Os dados foram analisados ao ozônio sobre a função longo do tempo de exposição e pulmonar do grupo pelo nível de exposição de saudável. ozônio em cada ponto de tempo para revelar relações dose-resposta mais estreitamente.

Modelo de Cobaias: porquinhos A exposição a SO2Conclui-se a partir destes Com a os testes de exposição exposição da Índia $\quad(0,1-16,6 \mathrm{ppm})$ foi resultados que a exposição verificou-se que o grau de repetida realizada numa $\mathrm{SO} 2$ em concentrações obstrução brônquica foi câmara de exposiçãobaixas e médias pode significativamente maior no de $20 \mathrm{G}$, durante 8 facilitar a sensibilização grupo exposto, em comparação horas em 5 diasalérgica local na cobaia. com o grupo de controle, para consecutivos, enquanto que a temperatura, umidade, e a concentração de $\mathrm{SO} 2$ foram monitorados e mantidos constantes. todas as concentrações de $\mathrm{SO} 2$ $(\mathrm{p}<0,05)$. Os anticorpos específicos para a OA no soro e no fluído broncoalveolar aumentaram nos grupos expostos-SO2

significativamente, em comparação com o grupo de controle $(\mathrm{p}<0,05)$.

402 crianças, em 5 Sensibilização foi As razões de prevalência Os dados sugerem uma relação países europeus. avaliada pela medição do ácaro ficaram entre dose-resposta em forma de sino do alérgeno específico média e alta quando entre a exposição ao alérgeno Imunoglobulina Ecomparada com os baixos do ácaro e sensibilização a contra ácaros. níveis de alérgenos. estes. Em populações com altos níveis de agentes microbianos e baixos índices de sensibilização, a curva é deslocada para baixo.

1386 sujeitos, (655Espirometria e testes Maiores taxas deNeste período de 10 anos, homens e $731 \mathrm{com}$ metacolina foram prevalência no diagnóstico houve aumento de asmáticos, mulheres) e uma realizados segundo ode asma detectado; mas a pelas mesmas causas do idade média de 41 protocolo. taxa de sintomas período de análise anterior. anos. Mudanças na prevalência após um período de 9 a 10 anos permanece semelhante e consistente de que mais pessoas estão classificadas como asmáticos.

Fonte: tabela elaborada pelo próprio autor com embasamento nos dados fornecidos pelos artigos científicos. 


\section{DISCUSSÃO}

Nos resultados obtidos por Castro et al (2002) em relação às crianças de sua amostra, foi proposta a "Teoria da Higiene", uma hipótese que explica a susceptibilidade da população urbana às alergias respiratórias, e que relaciona as melhorias das condições sócioeconômicas e as alterações da vida moderna com maior prevalência de doenças alérgicas, sustentada pelo artigo de Martinez (1994), Castro et al (2002), e ainda corrobora possível evidência a respeito de que o número de filhos possa contribuir para a expressão de atopia nas famílias. Autores como Stratchan (1997), Von Mutius (1994) e Martinez (1994), já demonstraram que o número de filhos é inversamente proporcional à prevalência de história pessoal de alergia respiratória, à reatividade no teste cutâneo e até à presença de anticorpos IgE específicos no soro. A maioria destes relatos prevê uma associação maior com o número de irmãos mais velhos do que com irmãos mais novos. Segundo Stratchan (1989), os irmãos mais novos estariam mais sujeitos as infecções nos primeiros anos de infância transmitidas via contato sem higiene através dos irmãos mais velhos, o que preveniria assim o desenvolvimento de atopia. Com base nos dados de Castro et al (2002), o número de filhos foi maior em Atibaia, em um bairro da zona rural, onde realmente estas inferências se confirmam, apesar da necessidade de maior coleta de informações para comprovar que tal sensibilização é de fato menor em irmãos mais novos através de um perfil sócioeconômico que não conseguiu ser posto em prática. Sustentando ainda a hipótese associativa entre condições sócio-econômicas e níveis de poluição e doenças respiratórias, Albuquerque de Castro (2007) propõe que a exposição às partículas aéreas pode causar uma variedade de problemas de saúde. Numerosos estudos, dentre eles os de Hernández-Cadena et al (2000) e Sino et al (2006), ligam exposições de longo e curto prazo aos níveis de partículas a um aumento de internações hospitalares, atendimentos de emergência e mesmo morte por doenças cardíacas ou pulmonares e aos problemas de saúde em geral. No estudo de Albuquerque de Castro (2007), foram utilizados dados ambulatoriais, abordagem incomum no Brasil, para avaliar os efeitos na saúde da exposição a poluentes atmosféricos. A distribuição espacial da asma em crianças menores de 6 anos de idade mostrou que os bairros com condições sociais e ambientais precárias apresentaram altas taxas de asma proporcionais. Poluentes estão intimamente vinculados como aumento de internações e número de mortes, aumentando os custos agregados de países em desenvolvimento. Um estudo prospectivo proposto por Sino et al (2006) em três cidades da América Latina (Cidade do México, Santiago-Chile, e São Paulo-Brasil) dá uma perspectiva sobre 
custos, poluição atmosférica e internações: nos próximos 20 anos, os efeitos da poluição do ar serão responsáveis por 156 mil mortes, quatro milhões de casos de asma e consultas de 300.000 médicos para crianças, com um custo que varia 21-165 bilhões de dólares. Schram-Bijkerk et al (2006) demonstra que os resultados encontrados com seu trabalho não estão de acordo com estudos de coorte anteriores, os quais demonstraram relação dose-resposta linear entre os níveis de alérgenos de ácaros e sensibilização ácaro em crianças na Alemanha e no Reino Unido. Isso pode estar relacionado a diferenças nos níveis de alérgenos ou diferenças nas populações estudadas. Tanto o estudo alemão quanto inglês consistiram, ao menos em parte, de crianças consideradas em risco especial de desenvolver alergia, por causa de alergia ou asma em seus pais. Para as crianças com uma história familiar, foi observada uma associação positiva entre os níveis de alérgenos de ácaros e sensibilização ácaro, enquanto o oposto foi encontrado para as crianças sem história parental. Em contraste, o estudo de Schram-Bijkerk (2006) mostrou relações de dose-resposta semelhantes para as crianças com e sem asma parental e/ou febre do feno. Aqueles estudos mostraram crescente risco contínuo de sensibilização com o aumento dos níveis de alérgenos de ácaros do pó da casa. Sugeriu-se que a alta exposição ao alérgeno gato pode produzir uma forma de tolerância por uma modificação na resposta
Th2 com produção de IgG4 específica. Estes estudos sugerem que esse mecanismo, se existir, pode também aplicar-se a níveis de alérgenos de ácaros, dependendo do nível de alérgenos e outros fatores modificadores possíveis, como alergia ou asma parental.

Sunyer et al (1997) avaliou a associação da prevalência da asma em adultos jovens com fatores de susceptibilidade e exposição ambientais, tendo em conta a idade de início da asma em cinco áreas de Espanha: Albacete, Barcelona, Galdakao, Huelva, e Oviedo, e baseado no quadro do Inquérito Europeu sobre Saúde Comunitária Respiratória (ECRHS). Foram considerados fatores de análise: Atopia à perenização (odds ratio (OR) $=10,2,95 \%$ intervalo de confiança 4,2-25) e alérgenos sazonais (11,5, 4,6-28), asma parental $(4,5,2,5-8,4)$, e ordem de nascimento. Histórico de asma foi associado a um menor grau, com atopia (OR em torno de 3,5 para ambos, perene e alérgenos sazonais). Infecções do trato respiratório inferior antes da idade de cinco anos (ITRI), tendo tido um animal de estimação na infância, e ter nascido em uma coorte mais jovem foram associados com asma antes da idade de 15 anos, mas não depois e o sexo masculino foi mais frequente na asma infantil enquanto o sexo feminino em idade adulta. Os fatores de risco conhecidos fornecem evidências de uma associação entre asma e a sensibilização aos alérgenos sazonais e o fato de se ter menos de três irmãos mais velhos, a 
uma associação de asma na infância com infecções do trato respiratório inferior.

Novamente, desta vez orquestrado pela equipe de Urrutia et al (2007), foi demonstrado, em continuidade com o estudo de Sunyer et al (1997), por um estudo de coorte de adultos jovens espanhóis, o aumento da prevalência e tratamento da asma, sem aumento de sintomas respiratórios. Este padrão permanece como há dez anos atrás, ainda que a hiperreatividade brônquica esteja incluída neste novo estudo. Estes resultados estão de acordo com a análise da seção europeia da ECRHS-II e são consistentes com a noção de que o atual aumento na consciência dos sintomas de asma, diagnóstico e tratamento de adultos é conseqüência da educação, de maior compreensão dos sintomas, e/ou mais rápida prescrição de medicamentos, e da maior disponibilidade de médicos para fazer $o$ diagnóstico e tratamento da doença.

Strand et al (1998), em seu estudo, demonstra que uma concentração ambiente de $\mathrm{NO}_{2}$ aumenta a resposta asmática da dosagem de alérgeno, porém por si só não causa reação asmática, porque o efeito só se reproduz após exposição repetida em laboratório. Contudo, os resultados laboratoriais permitem dizer que concentrações de $\mathrm{NO}_{2}$ podem afetar os asmáticos em situações reais e cotidianas, como é também corroborado por Rak et al (1988) Ihre e Zetterström (1993).

Sacramento de Oliveira (2011) descreve as alterações fisiológicas do envelhecimento a fim de explicar como os idosos são mais susceptíveis aos efeitos de PM10, porque todos os componentes do sistema respiratório são normalmente afetados pelo envelhecimento, incluindo: capacidade de difusão de oxigênio, elasticidade do pulmão, do tórax, capacidade de expansão da parede torácica, força muscular respiratória, consumo máximo de oxigênio, e do pico do débito cardíaco (OMS, 2006). Os idosos também estão mais propensos às infecções respiratórias, devido a um declínio relativo à idade na resposta imune específica, a função ciliar, e do reflexo de tosse. (Martins et al, 2002).

Em Utell et al (1994) e Riedel et al (1988), observaram-se no grupo de controle constituído por amostras saudáveis, sendo o primeiro de humanos e o segundo de porquinhos da Índia, foi percebido que não houve sensibilização significativa após a exposição ao $\mathrm{H}_{2} \mathrm{SO}_{4}$ e depois ao ozônio $\left(\mathrm{O}_{3}\right)$ e ao $\mathrm{SO}_{2}$, respectivamente. Contudo, para aqueles já diagnosticados com problemas respiratórios, houve sensibilidade suficiente para que houvesse aumento significativo no grau de obstrução brônquica.

Em suma, foram considerados fatores ambientais, sociais e biológicos nesta análise, a qual pretendeu mostrar como os fatores externos têm completa relação com o desenvolvimento das patologias respiratórias. 


\section{CONCLUSÃO}

$\mathrm{O}$ alcance da pesquisa realizada permite a avaliação de populações com diversas culturas e hábitos de vida, beneficiados ou não pelas questões ambientais. Mas o grande impacto e mérito deste trabalho diz respeito à correlação entre a hereditariedade das alergias respiratórias e também a influência da ordem de nascimento dos filhos para determinação destas doenças, além da susceptibilidade a tais patologias, intimamente relacionadas com determinantes socioeconômicos, culturais e biológicos. A contribuição para literatura científica está no favorecimento da rapidez na busca sobre um tema cotidiano, porém pouco

\section{REFERÊNCIAS BIBLIOGRÁFICAS}

CASTRO, F.F.M. et al. Sensibilization to aeroallergerns in schoolchildren living in rural and urban areas in São Paulo, Brazil. Rev.

\section{Brasileira de Alergia e Imunopatologia,}

São Paulo, Brasil. v.25 n.1, p. 02-09, fev, 2002. Disponível em:

$<$ http://www.asbai.org.br/revistas/Vol251/sen si.htm> . Acesso em: 12 nov. 2014.

CASTRO, H.A. et al. Air pollution and respiratory diseases in the Municipality of Vitória, Espírito Santo State, Brazil. Cad. Saúde Pública, Rio de Janeiro, Brasil. v.23 (supl. 4) p. S630-S642, 2007. Disponível em: <http://dx.doi.org/10.1590/S0102- abordado no meio acadêmico, que pode auxiliar muito na área prática da clínica médica, orientando modificações necessárias no ambiente e no modo de vida da população em estudo, além do investimento maior em políticas públicas que viabilizem o combate aos agentes poluidores da atmosfera e desencadeadores de doenças respiratórias.

Para novos estudos que sigam a mesma linhagem temática, seria prudente sugerir menor abrangência populacional e de região, e foco mais definido em determinada patologia respiratória, o que permitiria a continuidade do estudo promovido por este artigo, dessa vez sob uma óptica mais concisa e direcionada.

311X2007001600023>. Acesso em: 12 nov. 2014.

HERNÁNDEZ-CADENA, L. et al. Relación entre consultas uma urgencias por enfermedad respiratoria y contaminación atmosférica en Ciudad Juárez, Chihuahua. Salud Pública de Méx, Chihuahua, México. v. 42 n. 4 p. 28897, 2000. Disponível em: $<$ http://www.redalyc.org/articulo.oa?id=1064 2405>. Acesso em: 12 nov. 2014.

IHRE, E. ZETTERSTROM, O. Increase in non-specific bronchial responsiveness after repeated inhalation of low doses of allergen. Clin Exp Allergy, Stockholm, Sweden. v. 23 n. 4 p. 298-305, 1993. Disponível em: 
<http://www.ncbi.nlm.nih.gov/pubmed/83191

27>. Acesso em: 12 nov. 2014.

MARTINEZ, F.D. Role of viral infections in the inception of asthma and allergies during childhood: could they be protective? Thorax, Arizona, USA v. 49 p. 1189-1191, 1994.

Disponível em:

$<$ http://thorax.bmj.com/content/49/12/1189.fu 1l.pdf+html> . Acessado em: 07 dez. 2014.

MARTINS, L.C. et al. Poluição do ar e atendimentos por pneumonia e gripe em São Paulo, Brasil. Rev Saúde Pública, v. 36 p. 88-94, 2002. Disponível em: $<$ http://www.revistas.usp.br/rsp/article/view/2 5307>. Acesso em: 07 dez. 2014.

OLIVEIRA, M.S. et al. Differential susceptibility according to gender in the association between air pollution and mortality from respiratory diseases. Cad. Saúde Pública, Rio de Janeiro, Brasil. 27 (9), 1827-1836. Set, 2011. Disponível em: $<\mathrm{http}$ //dx.doi.org/10.1590/S0102311X2011000900016>. Acesso em: 12 nov. 2014.

\section{ORGANIZAÇÃO MUNDIAL DA}

SAÚDE. Diretrizes de qualidade de ar atualização global de 2005. Rheinbach: Organização Mundial de Saúde; 2006.

Disponível em:

<http://www.who.int/phe/health_topics/outdo orair/outdoorair_aqg/en/> . Acesso em: 07 dez. 2014.

RAK, S; LOWHAGEN, O; VENGE, P. The effect of immunotherapy on bronchial hyperresponsiveness and eosinophil cationic protein in pollen allergic patients. J Allergy Clin Immunol, Västerås, Gothenburg. v. 82 p. 470-480, 1988. Disponível em: <http://www.ncbi.nlm.nih.gov/pubmed/31709 95>. Acesso em: 07 dez. 2014.

RIEDEL, F. et al. Effects of SO2 exposure on allergic sensitization in the guinea pig. The

\section{Journal of Allergy and Clinical}

Immunology, Denver, Estados Unidos. v. 82 n.4 p. 527-534. Out, 1988. Disponível em: <http://www.jacionline.org/article/00916749(88)90961-X/abstract>. Acesso em: 12 nov. 2014.

SCHRAM-BIJKERK, D.G. et al. Nonlinear relations between house dust mite allergen levels and mite sensitization in farm and nonfarm children. Allergy: European

\section{Journal of Allergy and Clinical}

Immunology, Oxford, Reino Unido. v. $61 \mathrm{n}$. 5 p. 640-647. Mai, 2006. Disponível em: $<$ http://onlinelibrary.wiley.com/doi/10.1111/j. 1398-9995.2006.01079.x/abstract>. Acesso em 12 de nov. 2014.

SINO, M.L. et al. Os efeitos na saúde evitáveis de poluição do ar em três cidades latino-americanas: Santiago, São Paulo e 
Cidade do México. Environ Res v. 100 p. 431-440, 2006.

STRAND, V. et al. Repeated Exposure to na ambient level of NO2 enhances asthmatic response to a nonsymptomatic allergen dose.

European Respiratory Journal, Barcelona, Espanha. v.12 n.1 p. 06-12, Jul, 1998.

Disponível em:

<http://www.ncbi.nlm.nih.gov/pubmed/97014 06>. Acessado em: 12 nov. 2014.

STRATCHAN, D.P. et al. Sibship size and self-reported inhalant allergy among adult women. Clin. Exp. Allergy, London, UK. v. 27, n. 2 p. 151-155, 1997. Disponível em: <http://www.ncbi.nlm.nih.gov/pubmed/90612 13>. Acesso em: $07 \mathrm{dez} .2014$.

SUNYER, J. et al. Risk factors for asthma in young adults. European Respiratory

Journal, Barcelona, Espanha. v.10 n. 11 p. 2490-2494. Nov, 1997. Disponível em: $<$ http://erj.ersjournals.com/content/10/11/249 0.long>. Acesso em: 12 nov. 2014.

VON MUTIUS, E et al. Skin test reactivity and number of siblings. Br. Med. J., Munich, Germany. v. 12 n. 308 p. 692-695, 1994.
Disponível em: <

http://www.ncbi.nlm.nih.gov/pubmed/814279 3>. Acesso em: 07 Dez. 2014.

URRUTIA, I. et al. Changes in the prevalence of asthma in the Spanish cohort of the European Community Respiratory Health Survey (ECRHS-II). Archivos de Bronconeumología, Bizkaia, Espanha. v.43 n.8 p. 425-430. 2007. Disponível em: $<$ http://apps.elsevier.es/watermark/ctl_servlet ?_f=10\&pident_articulo=13109691\&pident_u suario $=0 \&$ pcontactid $=\&$ pident_revista $=260 \& \mathrm{t}$ $\mathrm{y}=147 \&$ accion $=$ L\&origen $=$ bronco $\& w e b=w w$ w. archbronconeumol.org \&lan $=$ en $\&$ fichero $=2$ 60v43n08a13109691pdf001.pdf>. Acesso em: 12 nov. 2014.

UTELL, M.J. et al. Oxidant and acid aerosol exposure in healthy subjects and subjects with asthma. Part II: Effects of sequential sulfuric acid and ozone exposures on the pulmonary function of healthy subjects and subjects with asthma. Research Report Health Effects Institute, Boston, Estados Unidos. n.70 p. 3793. Nov, 1994. Disponível em: <http://www.ncbi.nlm.nih.gov/pubmed/78485 87>. Acesso em: 12 nov. 2014. 